\title{
NUMERICAL INVESTIGATION OF FACTORS INFLUENCING THE EXPERIMENTAL DETERMINATION OF CONCRETE FRACTURE ENERGY
}

\author{
GILDA DAISSE $\grave{ }^{* \dagger}$, IOANNIS BOUMAKIS ${ }^{\dagger}$, CHRISTIAN CARLONI ${ }^{\dagger \dagger}$, \\ ROMAN WAN-WENDNER ${ }^{\dagger \dagger}$ \\ ${ }^{\dagger}$ Christian Doppler Laboratory, University of Natural Resources and Life Sciences \\ Vienna, Austria \\ e-mail: gilda.daisse@boku.ac.at \\ e-mail: ioannis.boumakis@boku.ac.at \\ ${ }^{\dagger \dagger}$ Case Western Reserve University, Department of Civil Engineering \\ Cleveland, OH USA \\ e-mail: cxc966@case.edu \\ ${ }^{\ddagger}$ Ghent University, Department of Structural Engineering \\ Ghent, Belgium \\ e-mail: roman.wanwendner@ugent.be
}

Key words: Strain rate, Fracture energy, Loading rate effect, LDPM

\begin{abstract}
The fracture energy is one of the most crucial parameters for the numerical investigation of damage propagation and failure in reinforced concrete members. The correct characterization of concrete fracture properties can be compromised by different laboratory limitations, such as specimens size, mode of control, loading rate and the test apparatus. Nowadays limited recommendations exist concerning the experimental evaluation of fracture energy for normal and high strength concrete. In order to investigate the differences between different specimen sizes, evaluate the effect of mode of control and analyze the influence of different set-up on the fracture test, a numerical analysis supported by an experimental campaign is presented. The Lattice Discrete Particle Model (LDPM) has been used to simulate concrete and to provide realistic crack patterns and crack widths. In the first part of the study the position of the traveling crack tip is identified with two approaches and then used to investigate the strain rate distribution along the ligament. It is well-known that concrete is a visco-elastic material with strain-rate dependent fracture properties. For this reason in the second part of the study the potential influence of differences in loading rate on the effective fracture energy determined by the work of fracture method is investigated with simulated three-point bending tests of differently sized specimens and two notch depths.
\end{abstract}

\section{INTRODUCTION}

The understanding and description of concrete fracture is of major importance for the safe design of structures [1]. The experimental evaluation of fracture energy is difficult for quasibrittle material and the results can be compro- mised by different laboratory limitations. In this work, the classical three-point bending configuration is adopted and some of the influence factors which may effect the determination of the fracture parameters are investigated by means of numerical simulations. Fracture 
tests on quasi-brittle material are often performed in crack mouth opening displacement (CMOD) control which ensures stable tests even for highly brittle specimens. Alternatively, fracture tests can also be controlled by loadpoint displacement if the load-frame is sufficiently stiff compared to the specimen compliance. Nowadays, it is widely known that concrete is a visco-elastic material with rate dependent fracture properties, but the possible implications of this behavior are still not fully studied. For this reason, in this work, three-point bending tests are simulated, replicating numerically both modes of control (open and closed loop). The strain and the strain rate at the cracktip are monitored throughout the entire fracture propagation process. The possible implication that the change of strain rate can introduce on the material are then investigated through simulations with three different loading rates: (1) the quasi-static rate, where the peak is reached in 320 seconds, (2) a loading rate one order of magnitude higher, and (3) a loading rate one order of magnitude lower. The results in terms of fracture energy and the peak-load are then presented and discussed. The well-known discrete concrete model, the Lattice Discrete Particle Model (LDPM) [2,3] is chosen with the extensions as discussed in [4,5]. The concrete creep effects are taken into account through a rate-type model based on the micro-prestresssolidification formulation, able to combine the different chemical and physical mechanisms.

\section{LDPM SIMULATIONS}

\subsection{Lattice Discrete Particle Model}

The LDPM is a concrete model able to simulate the mechanical interaction of coarse aggregate pieces embedded in a cementitious matrix. This meso-scale model is formulated in the framework of discrete element models, following the assumption of spherical aggregates, named particles. A lattice mesh is created around them, connecting all the centers of the aggregates. A system of polyhedral cells is created performing a Delauney tetrahedralization and domain tessellation on the concrete domain.
The cells interact with each other through triangular facets on which the vectorial constitutive law is acting. In the elastic range the normal stress is proportional to the normal strain through a parameter $E_{N}$ and the shear stresses are proportional to the shear strain through $E_{T}$, where $E_{N}=E_{0}$ ( $E_{0}=$ effective normal modulus) and $E_{T}=\alpha E_{0}$ ( $\alpha=$ shear-normal coupling parameter). When the elastic limit is reached, the LDPM formulation considers fracture and cohesion, pore collapse and friction. A more detailed description of the model can be found in [2,3].

\subsection{Creep Implementation}

In this contribution, creep is modeled in a rate-type form which is able to combine the different physical and chemical mechanisms. This form is based on the MicroPrestress-Solidification Theory (MPS) proposed by Bažant et al. [6, 7], where the total strain rate $\dot{\epsilon}_{t o t}$ is split into different strain rates that describe different physical mechanisms, as follows:

$$
\dot{\epsilon}_{t o t}=\dot{\epsilon}^{*}+\dot{\epsilon}_{\nu}+\dot{\epsilon}_{f}+\dot{\epsilon}_{s h}+\dot{\epsilon}_{t h}+\dot{\epsilon}_{d a m}
$$

where $\dot{\epsilon}^{*}$ is the instantaneous response, $\dot{\epsilon}_{\nu}$ is the viscoelastic response, $\dot{\epsilon}_{f}$ is the pure viscoelastic flow, $\dot{\epsilon}_{s h}$ and $\dot{\epsilon}_{t h}$ are respectively the hygral and thermal strain rate and $\dot{\epsilon}_{d a m}$ is the strain rate due to damage. For more details about the implementation of the adopted creep model one can refer to Boumakis et al. [4].

\subsection{Three Point Bending Model}

The aim of this study is to analyze some of the experimental factors which influence the experimental determination of fracture parameters. For this reason the classical threepoint-bending configuration is chosen, where a notched beam rests on two roller supports and is subjected to a concentrated load at its center. Three different geometries and two notch depths are modeled and investigated. The investigated dimensions are reported in Table 1 . 
Table 1: Geometry of three-point bending specimens.

\begin{tabular}{ccccc}
\hline Type & $\mathrm{D}[\mathrm{mm}]$ & $\mathrm{B}[\mathrm{mm}]$ & $\mathrm{L}[\mathrm{mm}]$ & $a_{0}[\mathrm{~mm}]$ \\
\hline A1 & 75 & 75 & 300 & $22.5(0.3 \mathrm{D})$ \\
$\mathrm{A} 2$ & 75 & 75 & 300 & $37.5(0.5 \mathrm{D})$ \\
$\mathrm{B} 1$ & 150 & 150 & 600 & $45(0.3 \mathrm{D})$ \\
$\mathrm{B} 2$ & 150 & 150 & 600 & $75(0.5 \mathrm{D})$ \\
$\mathrm{C} 1$ & 300 & 300 & 1200 & $90(0.3 \mathrm{D})$ \\
$\mathrm{C} 2$ & 300 & 300 & 1200 & $150(0.5 \mathrm{D})$ \\
\hline
\end{tabular}

\subsection{PID controller}

Three-point bending tests are typically performed with closed-loop control where the load applied on the top loading rate is controlled in such a way that a prescribed crack mouth opening displacement (CMOD) rate is ensured. In order to simulate this mode of control, also numerically a PID controller for this explicit solver is applied [8]. In general, a PID controller (proportional-integral-derivative controller) is a control loop feedback mechanism commonly used in industry. The algorithm continuously calculates an error value as the difference between a measured process variable and a desired set point, here the CMOD. The controller is then able to minimize the error over time by adjustment of a control variable, here the force at the load point.

\section{MATERIAL AND EXPERIMENTS}

This contribution is based on experimental results obtained by testing concrete of class $\mathrm{C} 25 / 50$. The mix design is reported in Table 2 .

Table 2: Concrete mix design.

\begin{tabular}{lc}
\hline Cement type & CEM II $42.5 \mathrm{~N}$ \\
Water to cement ratio, $w / c[-]$ & 0.63 \\
Cement content, $c\left[\mathrm{~kg} / \mathrm{m}^{3}\right]$ & 275 \\
$r_{\min } / r_{\text {max }}[\mathrm{mm}]$ & $4 / 16$ \\
Aggregate to cement ratio [-] & 7.59 \\
\hline
\end{tabular}

In order to adequately model the material, short-term and long-term properties are characterized.

\subsection{Short-term tests}

The material characterization performed consists of compression tests on cylinders and cubes, fracture tests and indirect tensile strength (Brazilian splitting) [9]. Compressive strength was determined using cubes with a side length of $150 \mathrm{~mm}$ and cylinders of dimensions $150 \times$ $300 \mathrm{~mm}$ and the tests were performed according to EN 12390-3 (2009). Tensile strength was determined through Brazilian splitting tests on cylindrical specimens with a diameter of $150 \mathrm{~mm}$ and a height of $70 \mathrm{~mm}$ according to DIN EN 12390-6:2000. The specimens were stored in water with added lime $(3 \mathrm{~g} / \mathrm{l})$ in a room with a constant temperature of $23^{\circ} \mathrm{C}$. The tests were performed at a concrete age of 70 days. The determined tensile strength, tensile strength, Young modulus and compressive strength of $3.52 \mathrm{MPa}, 31.1 \mathrm{GPa}$ and 36.8 / $36.45 \mathrm{MPa}$, respectively. In order to obtain the loading rate dependence on concrete, notched three point bending tests were carried out with five different rates (Table 3). The dimension of the specimens are $100 \times 100 \times 400 \mathrm{~mm}$ and the cut notch has a width of $4 \mathrm{~mm}$ and a depth of $30 \mathrm{~mm}$. All the fracture tests were performed in CMOD control and the load-point displacement was monitored by means of DIC measurements. The specimens tested with the quasi-static loading rate $(1 \mathrm{QS})$ reached the peak in about $320 \mathrm{~s}$.

Table 3: Three-point bending tests loading rates.

\begin{tabular}{ll}
\hline Relative rates & Tested rates \\
\hline $0.1 \mathrm{QS}$ & $0.01 \mu \mathrm{m} / \mathrm{s}$ \\
$0.3 \mathrm{QS}$ & $0.03 \mu \mathrm{m} / \mathrm{s}$ \\
$1 \mathrm{QS}$ & $0.1 \mu \mathrm{m} / \mathrm{s}$ \\
$3 \mathrm{QS}$ & $0.3 \mu \mathrm{m} / \mathrm{s}$ \\
$10 \mathrm{QS}$ & $1 \mu \mathrm{m} / \mathrm{s}$ \\
\hline
\end{tabular}

\subsection{Long-term tests}

Creep and shrinkage tests were performed on both sealed and drying cylinders with a diameter of $150 \mathrm{~mm}$ and a height of $300 \mathrm{~mm}$. The specimens were stored in a water bath saturated with lime solution until the desired age. Three strain gauges with $50 \mathrm{~mm}$ length were 
applied around the cylinder in a $120^{\circ}$ configuration. For creep tests four specimens were loaded in compression at around 30\% of the compressive strength. Two of the specimens were sealed using a vapor barrier in order to obtain two dataset for each basic and total creep. Also, sealed specimens were instrumented right after the demoulding of the concrete in order to measure autogenous shrinkage.

\subsection{Model Calibration}

The lattice discrete particle model is adopted to simulate the damage propagation. The static parameters are calibrated to match the above mentioned experimental results and they are reported in Table 4.

Table 4: Calibrated LDPM parameters.

\begin{tabular}{ll}
\hline Static Parameter & Value \\
\hline Effective Normal Modulus, $E_{0}$ & $50[\mathrm{GPa}]$ \\
Shear-Normal Coupling Parameter, $\alpha$ & $0.25[-]$ \\
Tensile Strength, $\sigma_{t}$ & $2.4[\mathrm{MPa}]$ \\
Tensile Characteristic Length, $\ell_{t}$ & $240[\mathrm{~mm}]$ \\
Shear Strength Ratio, $\sigma_{s} / \sigma_{t}$ & $3.5[-]$ \\
Initial Friction, $\mu_{0}$ & $0.2[-]$ \\
Softening Exponent, $n$ & $1.5[-]$ \\
\hline
\end{tabular}

\section{ANALYSIS OF THE INFLUENCE FACTORS}

The results shown in this section are the product of numerical simulations calibrated on the above introduced experiments.

\subsection{Mode of control}

Fracture tests involve three parameters: load, load-point displacement and crack mouth opening displacement (CMOD). During three-point bending tests the CMOD continuously increases as fracture proceeds and for this reason the crack opening has been proposed as control variable. This way of testing ensures the full conversion of the supplied energy into crack surface energy and it is the most used for brittle and quasi-brittle materials. However, laboratory limitations can lead to the necessity of per- forming the tests in constant displacement rate and a stable fracture propagation can be difficult to obtain. In this work, two modes of control are compared analyzing the strain rate at the actual crack-tip. The latter is determined with two different approaches and then compared. The first method for the determination of the cracktip position, is based on the assumption that the damage begins when the ultimate tensile strain of the concrete, $\epsilon_{T}$, has been exceeded (Fig. 1).

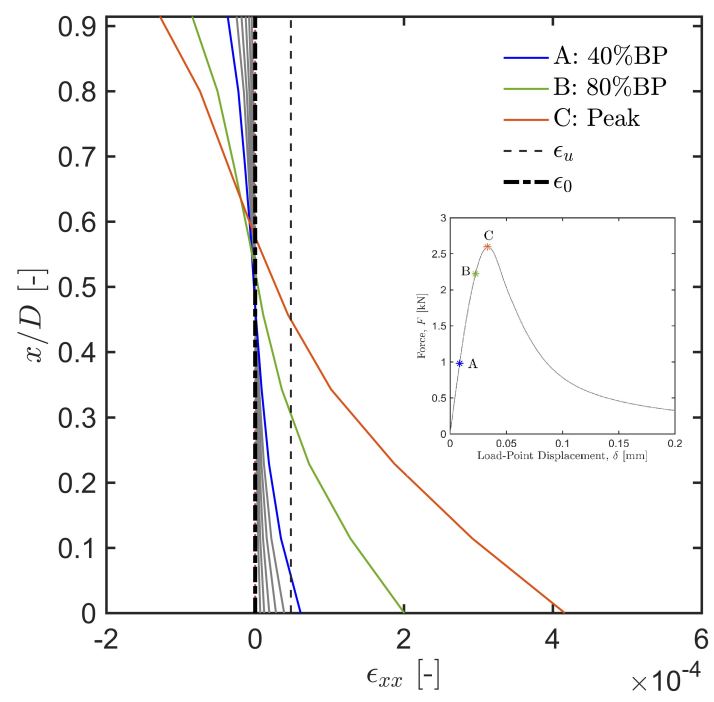

Figure 1: Strain profile of specimen A1 at different point in time: A) $40 \%$ before the peak, B) $80 \%$ before the peak and $\mathrm{C}$ ) at the peak.

To obtain this result, a set of virtual extensometers is applied on the specimen surface (Fig. 2 on the left) with a step of $6 \mathrm{~mm}$ between each of them, corresponding to the mesh size (1.5 coarse aggregate diameter). The second approach is based on the energy dissipated due to the fracture propagation. The relevant data are extracted from the LDPM simulations in the central region (Fig. 2 on the right) and then the dissipated energy is calculated for horizontal stripes of $6 \mathrm{~mm}$ height through a numeri- 
cal integration along the prism section [10]. The obtained value is the dissipated energy per unit area, which corresponds to the fracture energy for a fully softened traction free segment of a cohesive macro crack (Fig. 3).

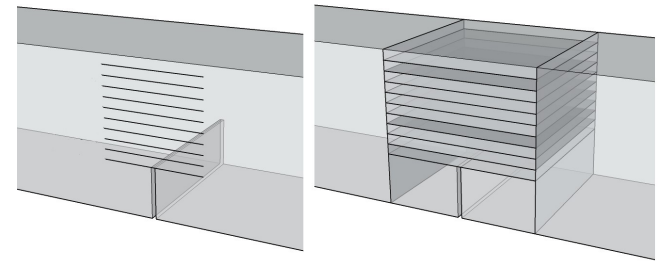

Figure 2: Left: schematized specimen geometry and virtual extensometer for the strain determination; right: schematized specimen geometry for the extrapolation of the dissipated energy.

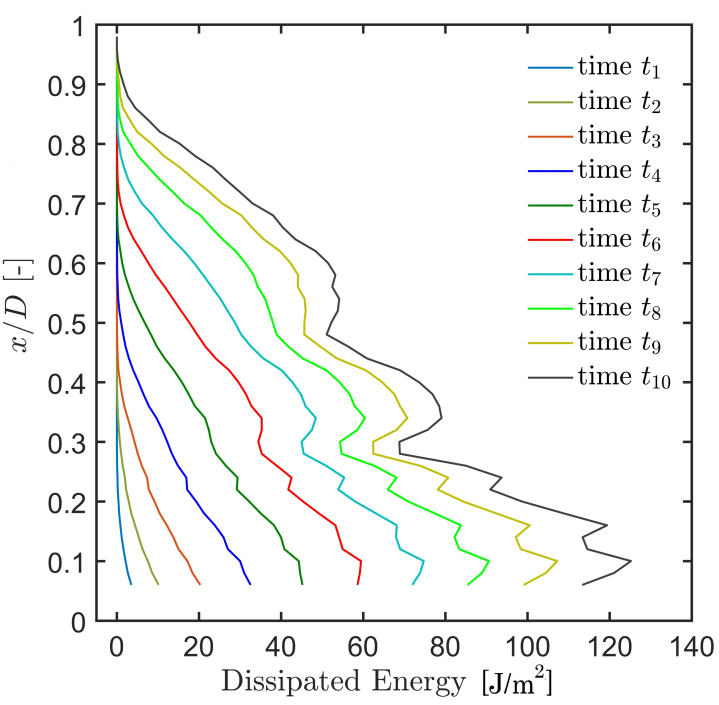

Figure 3: Dissipated energy along the ligament for different testing times.

Consequently, the crack-tip position can be defined as the point where the dissipated en- ergy exceeds a given minimum threshold, e.g. $1 \%$ of the fracture energy $G_{F}$. Both methods may have disadvantages. In the first case deformations measurements require the choice of base length for the engineering strain definition that needs to be large enough to contain the full width of the process zone but small enough to avoid averaging the localization zone over a too large area. The second approach is only available numerically and is biased by the discretization, here the distribution of LDPM cells. The determination of the position of the cracktip implies to establish two factors as indicated above: for the strain approach the length of the extensometer and for the energy approach the choice of the minimum fracture energy. Fig. 4 shows the position of the crack-tip over time, determined by the two methods.

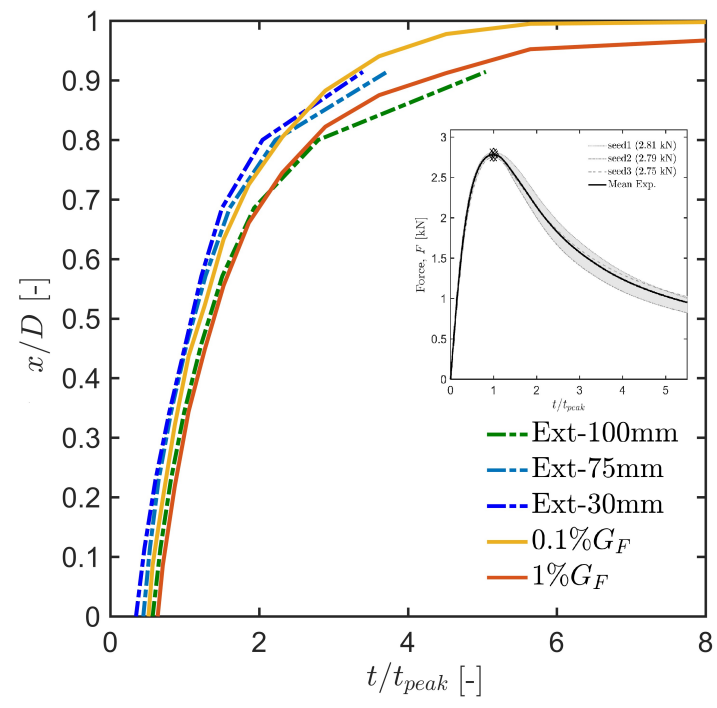

Figure 4: Comparison of the crack-tip position in time determined with the two approaches. The dashed lines are the results of the strain method using three virtual extensometer lengths $(30,75$ and $100 \mathrm{~mm})$. The solid lines are the output of the energy method considering two different energy thresholds $\left(0.1 \% G_{F}\right.$ and $\left.1 \% G_{F}\right)$. 
With the strain approach, three different virtual extensometer lengths (30, 75 and $100 \mathrm{~mm}$ ) were analyzed and for the second different fracture energy amounts are investigated $(0.1 \%$ and $\left.1 \% G_{F}\right)$.

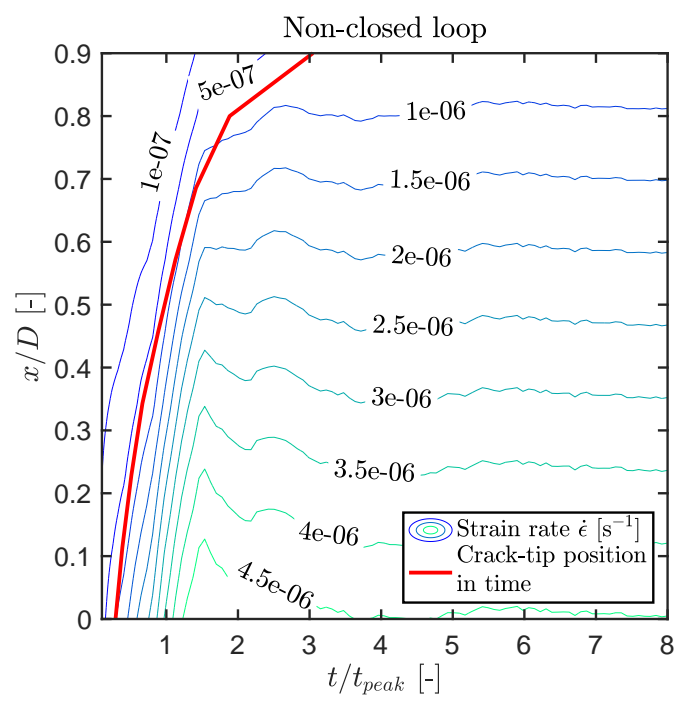

Figure 5: Strain rate at the crack-tip using a non-closed loop.

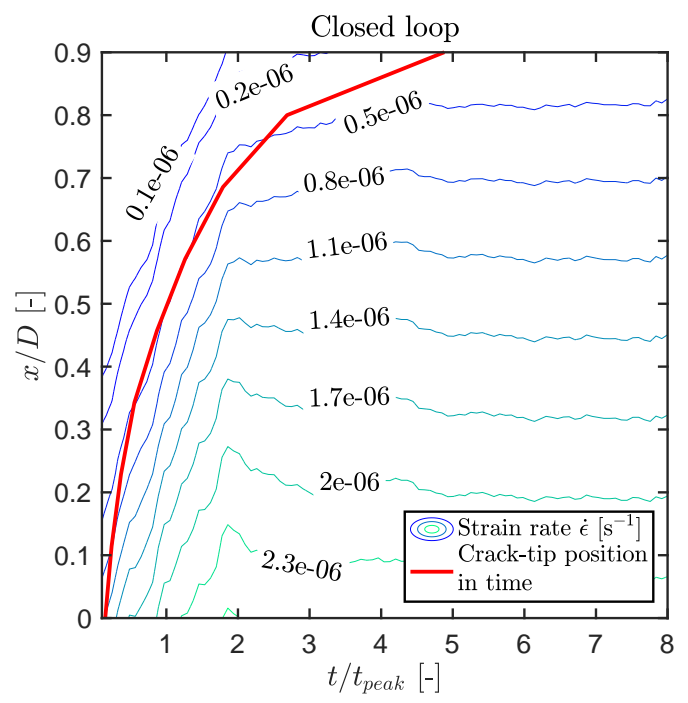

Figure 6: Strain rate at the crack-tip using a closed loop.

Due to the good degree of overlapping, the $100 \mathrm{~mm}$ extensometer has been chosen for the following strain rate analysis. The information about strain and time makes it easy to derive the strain rate. Knowing the exact position of the crack-tip, it is possible to see if its opening rate changes during its propagation along the ligament. In Fig. 5 and 6 the crack-tip position in time and the contour lines showing the strain rate are presented. The first one is the result of a simulation performed with a open loop (load point displacement control) while in the second one a closed-loop (CMOD control) is used. The test in displacement control takes less time for the complete propagation of the fracture and the strain rate is constant in almost all its development, only decelerating in the last phase. The test in CMOD control, on the other hand, show that the crack-tip strain rate decreases drastically in the first part, then becomes constant and finally decelerates again towards the end. In the range between 0 and 0.4 of the normalized ligament, the strain rate decreases of more then half of its speed (1.1e-6 to $0.5 \mathrm{e}-06 \mathrm{~s}^{-1}$ ), while the non-closed loop keeps a constant value of approximately $1 \mathrm{e}-6 \mathrm{~s}^{-1}$.

\subsection{Loading rate}

It is well known that the change of loading rate has a direct effect on the mechanical properties of concrete [11]. As discussed in section 2 , a rate-type model is implemented in this work in order to investigate the possible implication that the rate-dependency can have on the fracture parameters. Three-point bending simulations were run with three different loading rates, corresponding to the relative rates $0.1 \mathrm{QS}, 1 \mathrm{QS}$ and 10 QS shown in Table 3, where the quasistatic is the test velocity in which the peak is reached in 320 seconds. The rate effect implementation is calibrated on the experiments described in section 3.1. For each specimen geometry, the fracture energy and the maximum force reached are analyzed in relation to the test velocity. As widely known, the increase of the loading rate leads to an apparent strength increase (Fig. 8) which seems to be more accentuate in larger specimens. The consideration of the creep contribution accentuates the loading rate effect and decreases significantly the strength, as shown in Fig. 7. It is interesting 
to observed that the fracture energy at higher loading rates decreases as the rate gets slower (Fig. 9).

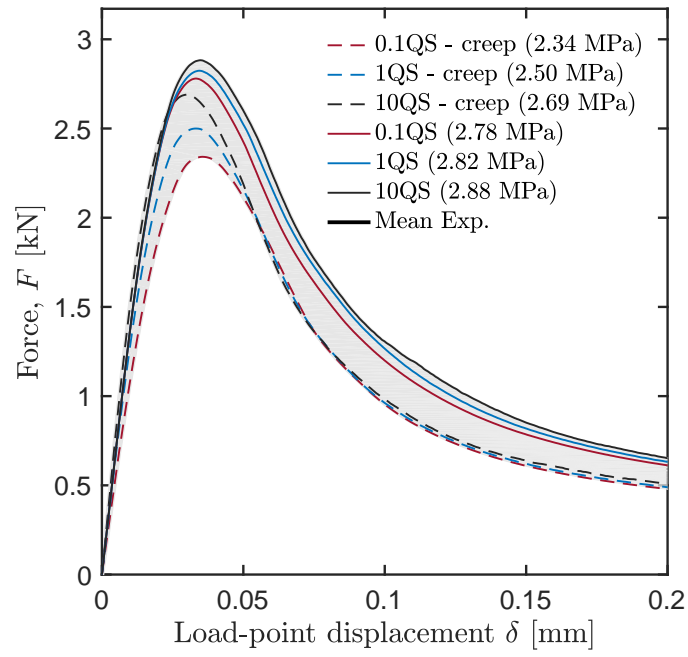

Figure 7: Force vs. load-point displacement curve for different loading rates. The dashed lines are the simulations with the creep contribution, while the solid lines are the simulations where only the rate effect is applied.

However, considering the creep contribution, small specimens showed that for very slow rates, the fracture energy seems to increase as the rate decreases (Fig. 9 on the right) as already observed experimentally by Wittmann et al. [12].
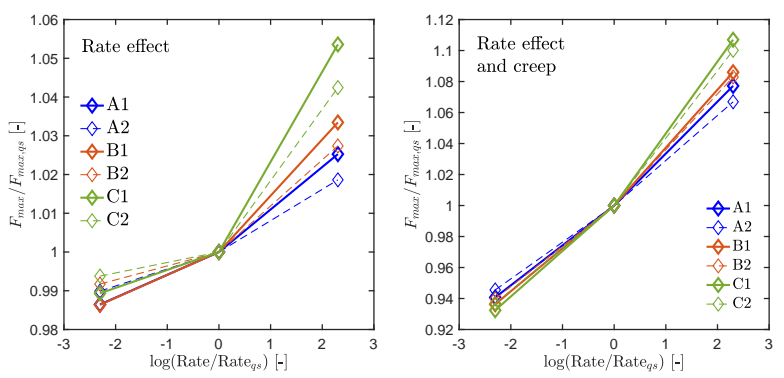

Figure 8: Loading rate effect on strength for the different specimens geometry
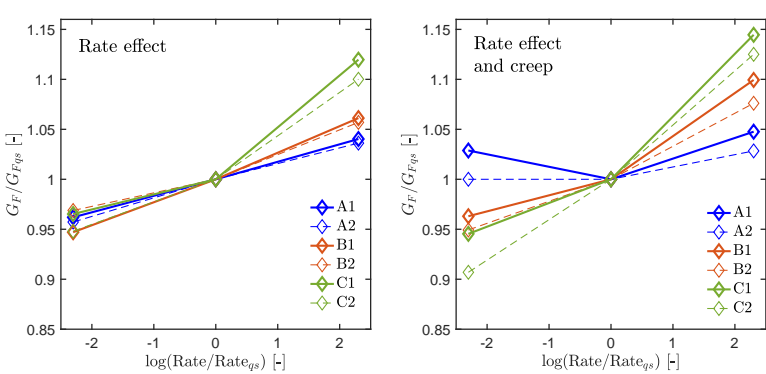

Figure 9: Loading rate effect on the fracture energy $G_{F}$ for the different specimens geometry

\section{CONCLUSIONS}

This work aims to analyze the influence of the laboratory conditions and limitations on the fracture properties. After simulating different test features on a three-point bending test, the following conclusions can be drawn:

- The different mode of control of fracture tests lead to different fracture propagation. The tests in displacement control have the advantage of maintaining a constant strain rate at the crack-tip but involve snap-back and instability problems, which makes the fracture energy determination difficult. However, the tests in CMOD control enable the stable fracture propagation, allowing the determination of the fracture energy, but it compromises the strain rate of the crack-tip, which decreases during test duration;

- Larger specimens are more influenced by the loading rate effect, both in terms of strength and fracture parameters.

- Creep has an important effect on fracture tests. It is well known that increasing the loading rate the strength increases and it has been observed that the creep contribution amplifies this phenomena.

- Small specimens could be more affected by the creep for very slow loading rates, showing a growth of the fracture energy when the rate slows down considerably. 


\section{ACKNOWLEDGEMENT}

The financial support by the Austrian Federal Ministry of Economy, Family and Youth and the National Foundation for Research, Technology and Development is gratefully acknowledged, as is the additional support by our industrial partners. The computational results presented have been achieved using the Vienna Scientific Cluster (VSC).

\section{REFERENCES}

[1] Bažant, Z.P., and Planas, J. 1998. Fracture and Size Effect in Concrete and Other Quasi-Brittle Materials, CRC Press LLC, Boca Raton.

[2] Cusatis, G., Pelessone, D., Mencarelli, A. 2011. Lattice Discrete Particle Model (LDPM) for failure behavior of concrete. I:Theory., Cement and Concrete Composites, 33, 881890.

[3] Cusatis, G., Mencarelli, A., Pelessone, D., 2011. Lattice Discrete Particle Model (LDPM) for failure behavior of concrete. II: Calibration and Validation., Cement and Concrete Composites, 33,891-905.

[4] Boumakis, I., Di Luzio, G., Marcon, M., Vorel, J., Wan-Wendner, R., 2018. Discrete element framework for modeling tertiary creep of concrete in tension and compression, Engineering Fracture Mechanics 200 (2018) 263-282.

[5] Boumakis, I., Marcon, M., Nincevic, K., Czernuschka, L.-M., Wan-Wendner, R., 2018. Concrete creep and shrinkage effect in adhesive anchors subjected to sustained load, Engineering Structures 175, pp. 790805.
[6] Bažant, Z.P., Hauggaard, A.B., Baweja, S., Ulm F.-J., 1997. Microprestresssolidification theory for concrete creep $i$ : Aging and drying effects, Journal of Engineering Mechanics 123 (11)1188-1194.

[7] Bažant, Z.P., Hauggaard, A.B., Baweja, S., 1997. Microprestress-solidification theory for concrete creep ii: Algorithm and verification, Journal of Engineering Mechanics 123 (11)1195-1201.

[8] Vorel, J., Marcon, M., Pelessone, D., Cusatis, G., Wendner, R., 2016. PID Controller in Explicit Solver, 9th International Conference on Fracture Mechanics of Concrete Structures FraMCoS-9, V. Saouma, J. Bolander, E. Landis, Berkeley.

[9] Czernuschka, L.-M., Wan-Wendner, R., Vorel, J., 2018. Investigation of fracture based on sequentially linear analysis, Engineering Fracture Mechanics 202, pp. 7586.

[10] Wan-Wendner, L., Wan-Wendner, R., Cusatis, G., 2018. Age-dependent size effect and fracture characteristics of ultrahigh performance concrete, Cement and Concrete Composite 85, 67-82.

[11] Yon, J.-H., Hawkins, N.M., Kobayashi, A.S., 1992. Strain-Rate Sensitivity of Concrete Mechanical Properties, ACI Materials Journal 89.

[12] Wittmann, F.H., Roelfstra, P.E., Mihashi, H., Huang,Y.-Y., Zhang, X.-H., Nomura, $\mathrm{N}$., 1987. Influence of age of loading, water-cement ratio and rate of loading on fracture energy concrete, Materials and Structures, 103-110. 